\title{
Inulin-type fructan degradation capacity of interesting butyrate-producing colon bacteria and cross-feeding interactions of Faecalibacterium prausnitzii DSM $17677^{\top}$ with bifidobacteria
}

\author{
Frédéric Moens", Audrey Rivière, Marija Selak, Luc De Vuyst
}

From Genes and nutrition, is personalised nutrition the next realistic step?

Brussels, Belgium. 25 April 2014

\section{Background}

Inulin-type fructans have already been studied with respect to their stimulation of bifidobacteria and butyrateproducing colon bacteria, such as Anaerostipes caccae and Roseburia spp. However, much less is known about their effects on other butyrate-producing colon bacteria, such as Butyricicoccus pullicaecorum, Eubacterium spp. and Faecalibacterium prausnitzii and the interactions of these species with bifidobacteria. This study aimed at investigating the kinetics of inulin-type fructan degradation and organic acid and gas production by B. pullicaecorum DSM $23266^{\mathrm{T}}$, E. hallii L2-7, E. rectale CIP $105953^{\mathrm{T}}$, and F. prausnitzii DSM $17677^{\mathrm{T}}$ and the possible interactions between $F$. prausnitzii DSM $17677^{\mathrm{T}}$ and bifidobacteria.

\section{Materials and methods}

All butyrate-producing strains were studied during screening experiments (100-ml scale) and monoculture fermentations (1.5-l scale) in a medium for colon bacteria (MCB) containing either fructose, oligofructose, or long-chain inulin as an energy source, supplemented with acetate. Coculture fermentation experiments (1.5-1 scale) in MCB were performed with $F$. prausnitzii DSM $17677^{\mathrm{T}}$ and Bifidobacterium breve Yakult, Bifidobacterium adolescentis LMG 10734, Bifidobacterium angulatum LMG $11039^{\mathrm{T}}$ (oligofructose), and Bifidobacterium longum LMG 11047 (oligofructose or inulin as a substrate).

\footnotetext{
* Correspondence: fmoens@vub.ac.be Research Group of Industrial Microbiology and Food Biotechnology (IMDO), Faculty of Sciences and Bio-engineering Sciences, Vrije Universiteit Brussel, Pleinlaan 2, B-1050 Brussels, Belgium
}

\section{Results}

Butyricicoccus pullicaecorum DSM $23266^{\mathrm{T}}$ and E. hallii L2-7 degraded fructose only, resulting in the production of butyrate, $\mathrm{H}_{2}$ and $\mathrm{CO}_{2}$. Eubacterium rectale CIP $105953^{\mathrm{T}}$ produced lactate and butyrate as well as $\mathrm{H}_{2}$ and $\mathrm{CO}_{2}$ out of fructose and inulin-type fructans. Faecalibacterium prausnitzii DSM $17677^{\mathrm{T}}$ produced butyrate, formate, and traces of lactate, together with $\mathrm{CO}_{2}$ out of fructose, oligofructose, and inulin. Both oligofructose-consuming, butyrate-producing strains degraded all oligofructose fractions simultaneously, indicating an extracellular degradation mechanism. During coculture fermentation experiments, oligofructose (by all bifidobacteria, except for $B$. breve Yakult) and inulin (by B. longum LMG 11047) were converted into acetate, lactate, and formate. Faecalibacterium prausnitzii DSM $17677^{\mathrm{T}}$ was cross-fed on this acetate resulting in the production of its metabolites. However, only low amounts of butyrate were produced during the coculture fermentations with $B$. angulatum LMG $11039^{\mathrm{T}}$ and B. longum LMG 11047, since $F$. prausnitzii DSM $17677^{\mathrm{T}}$ did not manage to compete well for the oligofructose substrate in the presence of $B$. angulatum LMG $11039^{\mathrm{T}}$ and B. longum LMG 11047, and for the inulin substrate in the presence of B. longum LMG 11047.

\section{Conclusion}

Besides cross-feeding interactions between bifidobacteria and butyrate-producing colon bacteria, competition for the available inulin-type fructans between these colon bacteria may occur. As a result, fast degraders such as bifidobacteria are favoured compared to acetate-depending butyrate producers. 
doi:10.1186/2049-3258-72-S1-06

Cite this article as: Moens et al: Inulin-type fructan degradation capacity of interesting butyrate-producing colon bacteria and crossfeeding interactions of Faecalibacterium prausnitzii DSM $17677^{\top}$ with bifidobacteria. Archives of Public Health 2014 72(Suppl 1):06.

Submit your next manuscript to BioMed Central and take full advantage of:

- Convenient online submission

- Thorough peer review

- No space constraints or color figure charges

- Immediate publication on acceptance

- Inclusion in PubMed, CAS, Scopus and Google Scholar

- Research which is freely available for redistribution

Submit your manuscript at www.biomedcentral.com/submit
C) Biomed Central 